\title{
GLUTATIONA REDUZIDA NA LIPOPEROXIDAÇÃO DO SÊMEN EQUINO CRIOPRESERVADO
}

Érika Saltiva Cruz Bender ${ }^{1}$, Breno Fernandes Barreto Sampaio ${ }^{2}$, Bruno Gomes Nogueira ${ }^{3}$, Marcilio $\mathrm{Nichi}^{4}$, Carmem Estefânia Serra Neto Zúccari ${ }^{5}$

${ }^{1}$ Mestre pelo Programa de Pós-Graduação em Ciência Animal - Faculdade de Medicina Veterinária e Zootecnia da Universidade Federal de Mato Grosso do Sul

${ }^{2}$ Doutor pelo Programa de Pós-Graduação em Ciência Animal - Faculdade de Medicina Veterinária e Zootecnia da Universidade Federal de Mato Grosso do Sul

${ }^{3}$ Doutorando do Programa de Pós-Graduação em Ciências Veterinárias - Faculdade de Medicina Veterinária e Zootecnia da Universidade Federal de Mato Grosso do Sul

${ }^{4}$ Professor Doutor da Faculdade de Medicina Veterinária e Zootecnia da Universidade de São Paulo

${ }^{5}$ Professora Doutora da Faculdade de Medicina Veterinária e Zootecnia da Universidade Federal de Mato Grosso do Sul (carmem.zuccari@ufms.br), Campo Grande - Brasil

Recebido em: 08/09/2015 - Aprovado em: 14/11/2015 - Publicado em: 01/12/2015 DOI: http://dx.doi.org/10.18677/Enciclopedia_Biosfera_2015_171

\section{RESUMO}

Os procedimentos de manipulação do ejaculado podem aumentar o estresse oxidativo sofrido pelos espermatozoides, com consequente redução da sua longevidade. Estudos mostram que o processo de criopreservação induz a lipoperoxidação, o que suscita a realização de pesquisas que estabeleçam estratégias para reduzir os danos causados. O objetivo do experimento foi avaliar o efeito antioxidante da glutationa reduzida adicionada ao sêmen equino précongelação, nas concentrações de $3 \mathrm{mM}, 5 \mathrm{mM}$ e $7 \mathrm{mM}$, sobre os níveis de lipoperoxidação após a descongelação. Os ejaculados $(n=19)$ foram congelados em diluente a base de gema de ovo $\left(\right.$ Botucrio $\left.^{\circledR}\right)$. Foram analisadas as variáveis espermáticas: motilidade, morfologia, viabilidade/status acrossomal, atividade mitocondrial e nível de lipoperoxidação. As médias dos tratamentos foram comparadas pelo teste de $L S D$, em nível de $5 \%$ de significância. Quando comparada a ação das diferentes concentrações de GSH após a descongelação, não foi observado efeito protetor contra a lipoperoxidação, sendo que as concentrações de $5 \mathrm{mM}(45,3 \%$ vs. $61,6 \%)$ e $7 \mathrm{mM}(25,3 \%$ vs. $61,6 \%)$ causaram uma redução significativa da motilidade quando comparadas ao grupo controle, respectivamente. Conclui-se que as concentrações de glutationa reduzida testadas não conferiram proteção antioxidante durante o processo congelação/descongelação do sêmen equino, com as concentrações de $5 \mathrm{mM}$ e $7 \mathrm{mM}$ apresentando efeito deletério sobre a motilidade espermática.

PALAVRAS-CHAVE: antioxidante, espermatozoide, estresse oxidativo, ROS 


\title{
REDUCED GLUTATHIONE ON LIPID PEROXIDATION OF EQUINE CRYOPRESERVED SEMEN
}

\begin{abstract}
Ejaculate handling procedures increases oxidative stress suffered by the sperm, with a consequent reduction in their longevity. Studies show that cryopreservation induces lipid peroxidation, which demonstrate the necessity in conducting research to establish strategies to reduce damage. The objective of the experiment was to evaluate the antioxidant effect of reduced glutathione added to the pre-freezing equine semen at concentrations of $3 \mathrm{mM}, 5 \mathrm{mM}$ and $7 \mathrm{mM}$, on lipid peroxidation levels after thawing. Ejaculates $(n=19)$ were frozen in egg yolk extender (Botucrio $\left.{ }^{\circledR}\right)$. Sperm variables analyzed were, motility, morphology, viability / acrosome status, mitochondrial activity and level of lipid peroxidation. Treatment means were compared by LSD tests at $5 \%$ of significance. When compared the action of different concentrations of GSH after thawing, there was no protective effect against lipid peroxidation, whereas $5 \mathrm{mM}(45.3 \%$ vs. $61.6 \%)$ and $7 \mathrm{mM}(25.3 \%$ vs. $61.6 \%)$ concentrations, caused a significant reduction in motility compared to the control group, respectively. Therefore, it's concluded that reduced glutathione concentrations tested did not provide antioxidant protection to freeze/thaw cycle of equine semen, with $5 \mathrm{mM}$ and $7 \mathrm{mM}$ concentrations presenting deleterious effect on sperm motility.
\end{abstract}

KEYWORDS: antioxidant, oxidative stress, sperm, ROS

\section{INTRODUÇÃO}

As biotecnologias da reprodução animal têm se mostrado, na atualidade, fundamentais para o progresso genético dos sistemas de produção animal, incluindo-se nesse contexto a espécie equina, que vem adotando de forma crescente o uso das biotécnicas de multiplicação animal na rotina dos haras (BRINSKO et al., 2011).

A preservação do sêmen equino, pela refrigeração e congelação, vem reduzindo fronteiras, possibilitando que uma égua possa ser inseminada com 0 sêmen de um garanhão alojado à longa distância. A criopreservação ainda possibilita o armazenamento do sêmen por período indeterminado, podendo assim ser utilizado no caso do garanhão encontrar-se enfermo ou apresentar infertilidade adquirida e até mesmo após o óbito (OLIVEIRA et al., 2013).

No que se refere à criopreservação, durante os procedimentos de manipulação do ejaculado há aumento da concentração de cálcio intracelular, da geração de espécies reativas de oxigênio (ROS) e redução da capacidade antioxidante decorrente da remoção do plasma seminal. Tais eventos levam os espermatozoides a um estresse oxidativo exacerbado e, com isso, à capacitação prematura, tendo como consequência uma redução da longevidade (BALL et al., 2001; NEILD et al., 2005). Essas informações suscitam a realização de pesquisas que busquem estratégias para reduzir estes danos, visando obter melhor qualidade seminal, a qual irá se refletir em maior taxa de fertilidade.

Ao contrário do plasma seminal, a célula espermática possui pequena quantidade de antioxidantes, e dentre estes a GSH (L-y-glutamil-L-cisteinilglicina) apresenta um papel importante, sendo um tripeptídeo, encontrado em todas as células (MEISTER, 1994). O efeito antioxidante da GSH exógena sobre o sêmen 
criopreservado ainda está pouco elucidado, e são escassos os relatos para a espécie equina. Da mesma forma, ainda não está definida qual a concentração ideal a ser acrescida no diluente de criopreservação do sêmen equino. O objetivo do presente experimento foi avaliar o efeito antioxidante da GSH, adicionada ao sêmen equino pré-congelação, sobre os níveis de lipoperoxidação pós-descongelação.

\section{Animais}

\section{MATERIAL E MÉTODOS}

Foram utilizados 10 garanhões adultos da raça Quarto de Milha, de fertilidade comprovada, alojados em propriedades localizadas nas proximidades de Campo Grande/MS, Brasil, sendo colhidos dois ejaculados de cada reprodutor, à exceção de um animal do qual se obteve apenas um ejaculado.

\section{Colheita e transporte do sêmen}

O sêmen foi colhido com auxílio de manequim, através de vagina artificial modelo Botucatu ${ }^{\circledR}$ (Botupharma Biotecnologia Animal, Botucatu/SP, Brasil). Após a colheita o sêmen foi filtrado, para separação da fração gel, e diluído em meio à base de leite desnatado para obtenção de uma concentração de $50 \times 10^{6}$ espermatozoides $/ \mathrm{mL}$. Para o transporte até 0 laboratório, 0 material foi acondicionado em container (CP - NUNES et al., 2008) a uma temperatura de $5^{\circ} \mathrm{C}$.

\section{Congelação e descongelação do sêmen}

O sêmen diluído foi submetido à centrifugação em tubos cônicos de $15 \mathrm{~mL}$ a $600 \mathrm{G}$ por 10 minutos. O pellet foi ressuspendido em Botucrio ${ }^{\circledR}$ (Botupharma Biotecnologia Animal, Botucatu/SP, Brasil), para a obtenção de uma concentração final de $200 \mathrm{x}$ $10^{6}$ espermatozoides $/ \mathrm{mL}$. O volume final foi fracionado em quatro alíquotas contendo $500 \times 10^{6}$ espermatozoides, às quais foi acrescida a glutationa reduzida, sendo constituídos os seguintes tratamentos: C - controle, sem glutationa; GSH $3-3 \mathrm{mM}$ de GSH; GSH 5 - $5 \mathrm{mM}$ de GSH e GSH $7-7 \mathrm{mM}$ de GSH. O sêmen foi envasado em palhetas de $0,5 \mathrm{~mL}$, lacradas com álcool polivinílico. O processo de congelação foi realizado adotando-se um período de 20 minutos de refrigeração a $5^{\circ} \mathrm{C}$, seguidos por 20 minutos de exposição ao vapor de nitrogênio líquido e subsequente imersão no mesmo. Após este procedimento, as palhetas foram acondicionadas em raques e armazenadas em botijão criogênico a $-196^{\circ} \mathrm{C}$. À descongelação utilizou-se banhomaria a $37^{\circ} \mathrm{C}$ por 30 segundos.

\section{Momentos da avaliação seminal}

As avaliações do sêmen foram efetuadas nos momentos pós-colheita (PC) e pós-descongelação (PD). 


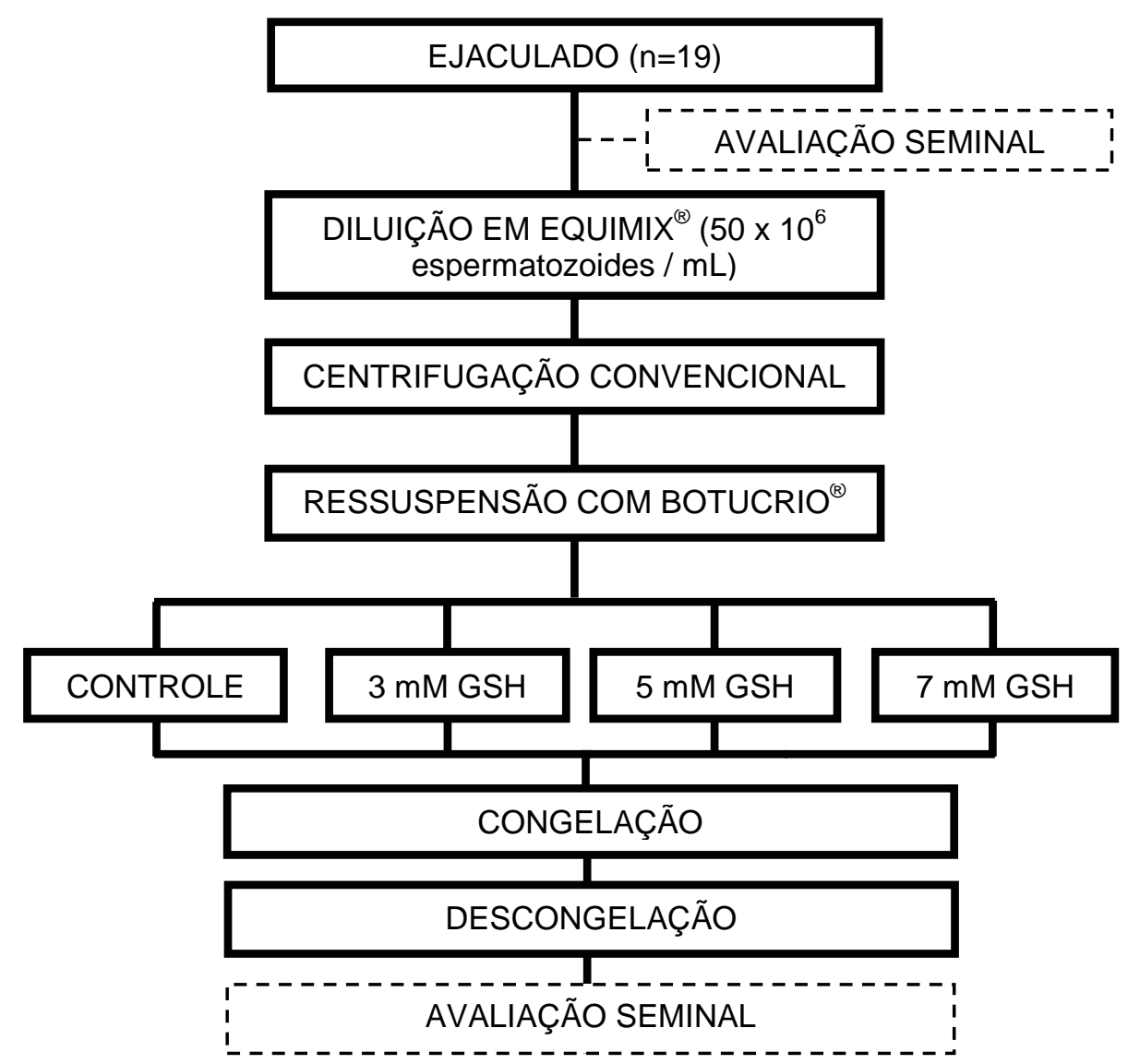

FIGURA 1. Fluxograma do protocolo experimental para avaliação dos efeitos da adição da glutationa reduzida (GSH) ao diluente de congelação, sobre o nível de lipoperoxidação no sêmen equino pósdescongelação.

\section{Motilidade}

A avaliação subjetiva da motilidade foi tipo cega, feita sob a microscopia de contraste de fase com objetiva de 40x, com uma gota de sêmen depositada entre lâmina e lamínula pré-aquecidas a $37^{\circ} \mathrm{C}$. A estimativa da motilidade foi feita pela determinação da proporção de espermatozoides móveis (0 a 100\%).

\section{Concentração espermática}

Para a estimativa da concentração espermática, $5 \mu \mathrm{L}$ de sêmen foram depositados em $95 \mu \mathrm{L}$ de água e a contagem feita com Câmara de Neubauer, através de microscopia de campo claro, com objetiva de 40x. O número médio de espermatozoides obtido, pela contagem de cinco quadrados em cada retículo, foi multiplicado por $1 \times 10^{6}$, o que resultou na determinação da concentração de espermatozoides / $\mathrm{mL}$.

\section{Morfologia espermática}

Amostras de sêmen destinadas à avaliação morfológica foram conservadas em solução de formol-salina-tamponada. Analisou-se 200 células/lâmina, em preparações úmidas, sob a microscopia de contraste de fase, em aumento de 1.000x. As patologias espermáticas foram classificadas de acordo com o proposto 
por BLOM (1973) citado por BARTH \& OKO (1989), e os resultados expressos em percentagem.

\section{Viabilidade e status acrossomal}

Para a avaliação simultânea da viabilidade e status acrossomal foi empregada a técnica de dupla coloração descrita por DIDION et al., (1989), que utiliza os corantes trypan blue / Giemsa. Os espermatozoides foram classificados em: vivos; mortos; reação do acrossomo verdadeira (RAV); reação do acrossomo falsa (RAF).

\section{Atividade mitocondrial}

$\mathrm{Na}$ determinação da atividade mitocondrial foi empregada a técnica descrita por HRUDKA (1987), que se baseia na oxidação da 3,3'- diaminobenzidina 4,4'tetraaminobiphenyl (DAB) pelo Complexo Citocromo C. As células foram classificadas em: DAB I - alta atividade mitocondrial; DAB II - média atividade mitocondrial; DAB III - baixa atividade mitocondrial, e DAB IV - sem atividade mitocondrial.

\section{Lipoperoxidação}

A avaliação foi feita de forma indireta, através da mensuração da concentração de espécies reativas ao ácido tiobarbitúrico (TBARS) induzida por vitamina C e Ferro, de acordo com NICHI et al., (2007). Os resultados foram expressos em nanogramas de MDA por $10^{8}$ de espermatozoides (ng/10 $0^{8} \mathrm{sptz}$ ).

\section{Análise Estatística}

Os dados obtidos foram analisados através do programa SAS System for Windows 9.3 (SAS, 2000). Através do aplicativo Guided Data Analisys, os dados foram testados quanto à normalidade dos resíduos (distribuição normal) e homogeneidade das variâncias. Caso não obedecessem a estas premissas, foram transformados (logaritmo na base 10 - Log10X; Raiz quadrada - RQ X; Quadrado X2) e se a normalidade não fosse obtida, empregava-se, então, o procedimento NPAR1WAY de análise de variância não paramétrica. Na avaliação do efeito do tratamento com GSH nas variáveis resposta pós-descongelação, foi utilizado o teste LSD (Least Significant Difference; comparações múltiplas). O nível de significância utilizado para rejeitar $\mathrm{HO}$ (hipótese de nulidade) foi de $5 \%$.

\section{RESULTADOS E DISCUSSÃO}

Após a colheita do sêmen a motilidade média foi $73,7 \pm 5,0 \%$, com 78,2 \pm $9,2 \%$ de espermatozoides vivos, $65,3 \pm 12,9 \%$ de células com alta atividade mitocondrial e nível de lipoperoxidação igual a 569,4 $\pm 573,8 \mathrm{ng} / 10^{8}$. À análise dos dados constatou-se que não houve interação significativa entre os momentos estudados e os tratamentos testados.

Após a descongelação foi observada uma redução da motilidade nos grupos em que foram adicionadas as concentrações de $5 \mathrm{mM}$ e $7 \mathrm{mM}$ de $\mathrm{GSH}(\mathrm{P}<0,05$; Tab.1). 
TABELA 1. Médias ( \pm erro padrão da média) das variáveis seminais dos ejaculados $(n=19)$ de garanhões Quarto de Milha, após a descongelação de acordo com as concentrações de glutationa reduzida adicionadas ao diluidor de congelação.

\begin{tabular}{|c|c|c|c|c|}
\hline \multirow{2}{*}{$\begin{array}{l}\text { Variáveis } \\
\text { seminais }\end{array}$} & \multicolumn{4}{|c|}{ Tratamentos } \\
\hline & Controle & GSH $3 \mathrm{mM}$ & GSH $5 \mathrm{mM}$ & GSH 7 mM \\
\hline Motilidade (\%) & $61,6 \pm 11,0^{a}$ & $55,8 \pm 13,9^{a}$ & $45,3 \pm 13,5^{b}$ & $25,3 \pm 16,1^{\mathrm{C}}$ \\
\hline Vivos (\%) & $51,8 \pm 13,1$ & $50,0 \pm 14,1$ & $49,8 \pm 12,4$ & $52,9 \pm 10,4$ \\
\hline DAB I (\%) & $37,2 \pm 17,3$ & $26,9 \pm 12,4$ & $25,4 \pm 9,9$ & $28,8 \pm 16,3$ \\
\hline TBARS [ng / & $874,2 \pm$ & $710,2 \pm$ & $636,3 \pm$ & $704,5 \pm 362,4$ \\
\hline $\left.10^{8}\right]$ & 854,8 & 529,0 & 348,9 & \\
\hline Variáveis & \multicolumn{4}{|c|}{ Tratamentos } \\
\hline seminais & Controle & GSH $3 \mathrm{mM}$ & GSH $5 \mathrm{mM}$ & GSH $7 \mathrm{mM}$ \\
\hline Motilidade (\%) & $61,6 \pm 11,0^{a}$ & $55,8 \pm 13,9^{a}$ & $45,3 \pm 13,5^{b}$ & $25,3 \pm 16,1^{\mathrm{C}}$ \\
\hline Vivos (\%) & $51,8 \pm 13,1$ & $50,0 \pm 14,1$ & $49,8 \pm 12,4$ & $52,9 \pm 10,4$ \\
\hline DAB I (\%) & $37,2 \pm 17,3$ & $26,9 \pm 12,4$ & $25,4 \pm 9,9$ & $28,8 \pm 16,3$ \\
\hline TBARS [ng / & $874,2 \pm$ & $710,2 \pm$ & $636,3 \pm$ & $704,5 \pm 362,4$ \\
\hline $\left.10^{8}\right]$ & 854,8 & 529,0 & 348,9 & \\
\hline Variáveis & \multicolumn{4}{|c|}{ Tratamentos } \\
\hline seminais & Controle & GSH $3 \mathrm{mM}$ & GSH $5 \mathrm{mM}$ & GSH $7 \mathrm{mM}$ \\
\hline Motilidade (\%) & $61,6 \pm 11,0^{a}$ & $55,8 \pm 13,9^{a}$ & $45,3 \pm 13,5^{b}$ & $25,3 \pm 16,1^{\mathrm{C}}$ \\
\hline Vivos (\%) & $51,8 \pm 13,1$ & $50,0 \pm 14,1$ & $49,8 \pm 12,4$ & $52,9 \pm 10,4$ \\
\hline DAB I (\%) & $37,2 \pm 17,3$ & $26,9 \pm 12,4$ & $25,4 \pm 9,9$ & $28,8 \pm 16,3$ \\
\hline TBARS [ng / & $874,2 \pm$ & $710,2 \pm$ & $636,3 \pm$ & $704,5 \pm 362,4$ \\
\hline $\left.10^{8}\right]$ & 854,8 & 529,0 & 348,9 & \\
\hline
\end{tabular}


A morfologia espermática não apresentou diferença significativa entre os tratamentos e os momentos avaliados. As concentrações de GSH testadas não demonstraram ação antioxidante durante 0 processo de criopreservação, apresentando, após a descongelação, valores de lipoperoxidação semelhantes ao controle. Esperava-se um menor nível de lipoperoxidação, induzida pela criopreservação, nas amostras acrescidas de GSH, preservando, desta forma, uma maior motilidade, atividade mitocondrial e viabilidade, o que não foi observado no presente trabalho. Isto sugere que a redução nos valores destas variáveis após a descongelação esteja relacionada, principalmente, às lesões induzidas pelo próprio processo de criopreservação, predominantemente causados pelo estresse osmótico (HAMMERSTEDT et al., 1990; POMMER et al., 2002).

Segundo ORTEGA-FERRUSOLA et al., (2009), um aumento da lipoperoxidação pós-descongelação, aparentemente, não é suficiente por si só para apresentar sinais evidentes de lesões induzidas pela criopreservação, mas pode desencadear alterações semelhantes a apoptose, as quais podem resultar em criodanos subletais nos espermatozoides vivos, levando ao envelhecimento precoce dos mesmos e consequente redução da sua vida útil após a descongelação. Estes danos subletais podem ter ocorrido no presente experimento, no entanto, não foi possível a detecção através das análises laboratoriais empregadas.

CÂMARA et al., (2011) levantaram a hipótese de que o diluente TRIS, formulado com gema de ovo in natura, teria uma significativa capacidade antioxidante, devido às lipoproteínas da gema do ovo, as quais são capazes de prevenir a oxidação dos ácidos graxos poli-insaturados - PUFAs (SAKANAKA et al., 2004). No presente experimento o diluente utilizado também apresenta gema de ovo na formulação, o que talvez possa ter mascarado os resultados, não se detectando diferença significativa entre os grupos quanto à lipoperoxidação. No entanto, a motilidade pós-descongelação dos grupos GSH 5 e GSH 7 foram significativamente menores, comparada ao controle e ao GSH 3, sugerindo algum efeito deletério destas concentrações.

Pesquisas sobre os efeitos da adição da GSH ao sêmen de mamíferos mostraram que a ação é dose-dependente, ou seja, pode não apresentar efeito, ser tóxica, ou, mostrar-se efetivamente protetora para as células (SINHA et al., 1996; GADEA et al., 2005, 2007; SILVA et al., 2011). A ação antioxidante desejada deve inativar continuamente os catabólitos resultantes do metabolismo oxidativo, de forma que se mantenha a quantidade de ROS necessária para a função fisiológica normal do espermatozoide, evitando assim, o chamado estresse redutivo (AGARWAL et al., 2003). Por este motivo, diferentes concentrações devem ser testadas até se determinar qual proporcionará a maior ação protetora possível.

Quando utilizada a concentração de $7 \mathrm{mM}$, foi observado um efeito tóxico, apresentando menor valor de motilidade $(P<0,05)$ após a descongelação, quando comparado aos demais tratamentos. Este efeito também foi observado por SILVA et al., (2011) para a mesma concentração $(7 \mathrm{mM})$, no sêmen ovino. Estes achados sugerem que se deve trabalhar com doses de GSH abaixo de $7 \mathrm{mM}$ para o sêmen equino e ovino, buscando-se atingir o efeito antioxidante desejado.

Por outro lado, BAUMBER et al., (2005) não observaram efeito tóxico, após a descongelação, testando $10 \mathrm{mM}$ em sêmen equino. Isto pode ter ocorrido devido às diferenças na metodologia, como a raça dos garanhões (Puro Sangue Inglês e Árabe) e a composição do diluente de criopreservação, o INRA 82 com adição de $2,5 \%$ de glicerol. O INRA 82 é um diluente à base de leite, enquanto o Botucrio ${ }^{\circledR}$, utilizado no presente experimento, tem como base a gema de ovo. No experimento 
de SILVA et al., (2011) também foi usado um diluente a base de gema de ovo (TRIS), o que leva a crer que, talvez, o efeito tóxico não seja resultado apenas da ação da $\mathrm{GSH}$, e sim de alguma reação entre os constituintes dos diluentes. No entanto, em estudo recente com adição de glutationa no sêmen equino refrigerado diluído em INRA 82, foi observada queda da motilidade progressiva e integridade de membrana plasmática quando adicionados $10 \mathrm{mM}$ do antioxidante após 24 horas de refrigeração (ZHANDI \& GHADIMI, 2014).

A GSH pode agir diretamente sobre as ROS, mas sua principal função é servir como substrato para a glutationa peroxidase (GPx), que catalisa a redução de peróxido de hidrogênio e hidroperóxidos tóxicos (MEISTER, 1994; BILODEAU et al., 2001). Como na espécie equina o plasma seminal é descartado ao final do processo de centrifugação, talvez se faça necessária a associação da GSH com a GPx, quando da adição ao diluente, potencializando a ação antioxidante. Outros estudos que também fizeram a remoção do plasma seminal no processo de criopreservação, não encontraram efeito significativo quando a GSH foi adicionada, seja em sêmen suíno (GADEA et al., 2004) ou equino (BAUMBER et al., 2005).

Em espécies em que não se faz a retirada do plasma seminal para a congelação, o efeito antioxidante da adição da GSH pré-congelação foi significativo (SINHA et al., 1996 - caprino; SILVA et al., 2011 - ovino) ou pós-descongelação (GADEA et al., 2007 - bovino). GADEA et al., (2005) observaram efeito significativo adicionando a GSH pré-congelação (1 e 5 mM) em sêmen suíno.

Recentemente, GIBB et al., (2014) apresentaram correlação positiva entre os níveis de ROS e a fertilidade do sêmen equino, no entanto estes resultados foram encontrados utilizando o sêmen fresco. Além disso, também relataram menor viabilidade espermática e integridade acrossomal em decorrência do aumento das ROS, o que pode afetar negativamente o sêmen quando armazenado.

Em síntese, as concentrações testadas de GSH não apresentaram efeito protetor contra a lipoperoxidação durante a criopreservação. Para se atingir uma máxima proteção, sem que sejam afetados os níveis fisiológicos de ROS, novos estudos são necessários, devendo ser avaliadas outras concentrações e o efeito da GSH frente a diferentes composições de diluentes, bem como sua associação com a glutationa peroxidase.

\section{CONCLUSÕES}

As concentrações de $3 \mathrm{mM}, 5 \mathrm{mM}$ e $7 \mathrm{mM}$ de glutationa reduzida não conferiram proteção antioxidante à descongelação do sêmen de garanhões, com as concentrações de $5 \mathrm{mM}$ e $7 \mathrm{mM}$ apresentando efeito deletério sobre a motilidade espermática.

\section{AGRADECIMENTOS}

Ao Conselho Nacional de Desenvolvimento Científico e Tecnológico (CNPq) pela concessão da bolsa de mestrado.

\section{REFERÊNCIAS}

AGARWAL, A.; SALEH, R.A.; BEDAIWY, M.A. Role of reactive oxygen species in the pathophysiology of human reproduction. Fertility and Sterility, v.79, p.829-843, 2003. 
BALL, B.A.; VO, A.T.; BAUMBER, J. Generation of reactive oxygen species by equine spermatozoa. American Journal of Veterinary Research, v.62, p.508-515, 2001.

BARTH, A.D.; OKO, R.J. Abnormal morphology of bovine spermatozoa. 1. ed. lowa State University Press: Ames, 1989, 285p.

BAUMBER, J.; BALL, B.A.; LINFOR, J.J. Assessment of the cryopreservation of equine spermatozoa in the presence of enzyme scavengers antioxidants. American Journal of Veterinary Research, v.66, p.772-229, 2005.

BILODEAU, J.F.; BLANCHETTE, S.; GAGNON, C.; SIRARD, M.A. Thiols prevent $\mathrm{H}_{2} \mathrm{O}_{2}$ mediated loss of sperm motility in cryopreserved bull semen. Theriogenology, v.56, p.275-286, 2001.

BRINSKO, S.P.; BLANCHARD, T.L.; VARNER, D.D.; SCHUMACHER J.; LOVE C.C.; HINRICHS K.; HARTMAN, D. Manual of Equine Reproduction, 3. ed. MOSBY Elsevier, cap.12, p.160-175, 2011.

CÂMARA, D.R.; SILVA, S.V.; ALMEIDA, F.C.; NUNES, J.F.; GUERRA, M.M.P.; 2011. Effects of antioxidants and duration of pre-freezing equilibration on frozenthawed ram semen. Theriogenology, v.76, p.342-350, 2011.

DIDION, B.A., DOBRINSKI, J.R., GILES, J.R., GRAVES, C.N., 1989. Staining procedure to detect viability and true acrosome reaction in spermatozoa of various species. Gamete Research, v.22, p.51-57, 1989.

GADEA, J.; GARCÍA-VASQUEZ, F.; MATÁS, C.; GARDÓN, J.C.; CÁNOVAS, S.; GUMBAO, D. Cooling and freezing of boar spermatozoa: supplementation of the freezing media with reduced glutathione preserves sperm function. Journal of Andrology, v.26, p.396-404, 2005.

GADEA, J.; GUMBAO, D.; CÁNOVAS, S.; GARCÍA-VÁSQUEZ, F.A.; GRULLÓN, L.A.; GARDÓN, J.C. Supplementation of the dilution medium after thawing with reduced glutathione improves function and the in vitro fertilizing ability of frozenthawed bull spermatozoa. International Journal of Andrology, v.31, p.40-49, 2007.

GADEA, J.; SELLÉS, E.; MARCO, M.A.; COY, P.; MATÁS, C.; ROMAR, R.; RUIZ, S. Decrease in glutathione content in boar sperm after cryopreservation: effect of the addition of reduced glutathione to the freezing and thawing extenders. Theriogenology, v.62, p.690-701, 2004.

GIBB, Z.; LAMBOURNE, S.R.; AITKEN, R.J. The paradoxical relationship between stallion fertility and oxidative stress. Biology of Reproduction, v.91, p.1-10, 2014.

HAMMERSTEDT, R.H.; GRAHAM, J.K.; NOLAN, J.P. Cryopreservation of mammalian sperm: what we ask them to survive. Journal of Andrology, v.11, p.7388, 1990. 
HRUDKA, F. Cytochemical and ultracytochemical demonstration of cytochrome c oxidase in spermatozoa and dynamics of its changes accompanying ageing or induced by stress. International Journal of Andrology, v.19, p.809-828, 1987.

MEISTER, A. Glutathione, ascorbate, and cellular protection. Cancer Research, v.54, p.1969-1975, 1994.

NEILD, D.M.; BROUWERS, J.F.H.M.; COLENBRANDER, B.; AGUERO, A.; GADELLA, B.M. Lipid peroxide formation in relation to membrane stability of fresh and frozen thawed stallion spermatozoa. Molecular Reproduction and Development, v.72, p.230-238, 2005.

NICHI, M.; GOOVAERTS, I.G.F.; CORTADA, C.N.M.; BARNABE, V.H.; DECLERCQ, J.B.P.; BOLS, P.E.J. Roles of lipid peroxidation and cytoplasmic droplets on in vitro fertilization capacity of sperm collected from bovine epididymis stored at 4 and $34 \stackrel{\circ}{\mathrm{C}}$. Theriogenology, v.67, p.334-340, 2007.

NUNES, D.B.; ZORZATTO, J.R.; COSTA E SILVA, E.V.; ZÚCCARI, C.E.S.N. Efficiency of short-term storage of equine semen in a simple-design cooling system. Animal Reproduction Science, v.104, p.434-439, 2008.

OLIVEIRA, G.C.; OLIVEIRA, B.M.M.; CELEGHINI, E.C.C.; FERNANDES, C.B.; MATTOS, C.B. Criopreservação do sêmen equino: uma revisão. Revista Brasileira de Reprodução Animal, v.37, n.1, p.23-28, 2013.

ORTEGA-FERRUSOLA，C.; GONZÁLVEZ FERNÁNDEZ, L.; MORRELL, J.M.; SALAZAR SANDOVAL, C.; MACÍAS GARCÍA, B.; RODRÍGUEZ-MARTINEZ, H.; TAPIA, J.A.; PEÑA, F.J. Lipid peroxidation, assessed with BODIPY-C ${ }_{11}$, increases after cryopreservation of stallion spermatozoa, is stallion-dependent and is related to apoptotic-like changes. Reproduction, v.138, p.55-63, 2009.

POMMER, A.C.; RUTLANT, J.; MEYERS, S.A. The role of osmotic resistance on equine spermatozoal function. Theriogenology, v.58, p.1373-1384, 2002.

SAKANAKA, S.; TACHIBANA, Y.; ISHIHARA, N.; JUNEJA, L.R. Antioxidant activity of egg-yolk protein hidrolysates in linoleic acid oxidation system. Food Chemistry, v.86, p.99-103, 2004.

SAS - Statistical Analysis System. SAS/STAT software, Version 9.3, 2000.

SILVA, S.V.; SOARES, A.T.; BATISTA, A.M.; ALMEIDA, F.C.; NUNES, J.F.; PEIXOTO, C.A.; GUERRA, M.M.P. In vitro and in vivo evaluation of ram sperm frozen in tris egg-yolk and supplemented with superoxide dismutase and reduced glutathione. Reproduction in Domestic Animals, v.46, p.874-881, 2011.

SINHA, M.P.; SINHA, A.K.; SINGH, B.K.; PRASAD, R.L. The effect of glutathione on the motility, enzyme leakage and fertility of frozen goat semen. Animal Reproduction Science, v.41, p.237-243, 1996.

ZHANDI, M.; GHADIMI, V. Effect of glutathione-supplemented INRA82 extender on miniature Caspian stallion sperm quality during storage at $5^{\circ} \mathrm{C}$. Journal of Equine Veterinary Science, v.34, p.606-610, 2014. 